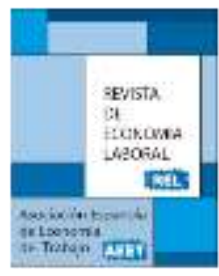

\title{
TRAYECTORIAS OCUPACIONALES DE LOS JÓVENES DEL BRASIL METROPOLITANO ¿INACTIVIDAD PARA ESTUDIAR O PARA SER NI-NI? (2002 - 2015) ${ }^{1}$
}

\author{
Sandro Eduardo Monsueto ${ }^{2 *}$, Mariangela Furlan Antigo ${ }^{* *}$, \\ Jaqueline Moraes Assis Gouveia*** y Anne Caroline Costa Resende*** \\ * Universidade Federal de Goiás, ${ }^{* *}$ Universidade Federal de Minas Gerais y ***Universidade Estatual de Campinas
}

Recibido Julio 2018; Aceptado Diciembre 2018

\section{Resumen}

El presente artículo tiene como objetivo analizar la evolución del tiempo medio en que los jóvenes brasileños permanecen en la inactividad laboral y si este tiempo está asociado a aumentos en la cualificación o con el fenómeno conocido como ni-ni. El análisis es realizado para el periodo entre 2002 y 2015, con los datos de la Pesquisa Mensal de Emprego (PME) del Instituto Brasileiro de Geografia e Estatística (IBGE). Los resultados parecen evidenciar un aumento del tiempo en lo cual los jóvenes permanecen como ni-ni en Brasil, sobre todo entre aquellos con edad entre 18 y 29 años. También revelan una dificultad de salida de esta condición, dada la dependencia temporal significativa.

Palabras clave: Jóvenes, Brasil, Generación ni-ni.

Clasificación JEL: J20, J22, J24

\section{Abstract}

This article aims to analyze the evolution of the average time in which young Brazilians remain in inactivity. In this way, we intends to considering if the behavior is associated with increases in qualification or with the phenomenon known as NEET. The analysis is performed for the period between 2002 and 2015, with the data of the Pesquisa Mensal de Emprego from Instituto Brasileiro de Geografia e Estatistica (IBGE). The results seem to show an increase in the time in which young people remain as NEET in Brazil, especially among those between 18 and 29 years old. They also reveal a difficulty of leaving this condition, given the significant temporal dependence.

Key Words: Young people, Brazil, NEET.

JEL Classification: J20, J22, J24

\footnotetext{
1 Trabajo financiado por la FAPEMIG (Convocatoria 01/2014 - proceso APQ 02764-14) y por la Convocatoria 02/2016 del Programa Pesquisador Mineiro - PPM X, Proceso PPM00658-16.

2 Correos de contacto: monsueto@ufg.br; maantigo@cedeplar.ufmg.br; jaquelinemagouveia@gmail.com; anneccr@gmail.com
}

(C) Revista de Economía Laboral 


\section{Introducción}

Desde la mitad de los años noventa del siglo pasado una variedad de investigaciones tiene analizado empíricamente la incidencia de un grupo de jóvenes que no trabajan y que, al mismo tiempo, no estudian, configurando lo que se conoce en Brasil como "generación ni-ni" (Camarano, 2006; DIEESE, 2008). Según datos de la Pesquisa Nacional por Amostra de Domicílios (PNAD), cerca de un 17\% de los jóvenes brasileños con edad entre 18 y 25 años se encuentran en esta condición en 2012. Este fenómeno tiene importantes implicaciones sociales y económicas para el país, puesto que, desde un punto de productivo, representa una generación de jóvenes que no está produciendo o acumulando conocimientos y tampoco realizando inversiones en capital humano (Borjas, 2008). Son individuos que, en el largo plazo, cuando se reincorporan al mercado del trabajo, se ven afectados por una baja acumulación de experiencia y, finalmente, impacta también en una baja acumulación de cotizaciones al sistema de pensiones que, como consecuencia, afecta su calidad de vida en la vejez. Desde el punto de vista social, pueden estar asociados a situaciones problemáticas, envolviendo comportamientos antisociales (Simmons, 2008).

Sin embargo, la mayor parte de la literatura empírica dedica espacio para la discusión estática de la situación en el país. A estos efectos, el presente artículo tiene como foco la determinación del tiempo en que se permanece como inactivo y fuera de la escuela. En otras palabras, el objetivo es analizar la evolución del tiempo medio que los jóvenes brasileños permanecen en la inactividad laboral y si este tiempo está asociado a aumentos en la cualificación o con el fenómeno "ni-ni”. El análisis es realizado para el mercado de trabajo metropolitano de Brasil entre 2002 y 2015, con los datos de la Pesquisa Mensal de Emprego, permitiendo capturar una parte de los efectos de la reciente crisis, entre jóvenes con edad entre 15 y 29 años. Se utiliza una matriz de transición de Markov para evaluar la probabilidad de transición para cada estado del mercado de trabajo y el porcentaje de tiempo en que permanece en esta condición, y un modelo logit para evaluar los determinantes de la entrada.

\section{Revisión bibliográfica}

La literatura acerca del tema de jóvenes que no trabajan y no estudian, toma como punto de partida los modelos tradicionales de 
búsqueda de empleo y de capital humano para entender el fenómeno y sus consecuencias. Layard, Nickell y Jackman (2005), por ejemplo, presentan un modelo para explicar la obtención de un puesto de trabajo en función de variables agregadas macroeconómicas, que reflejan el nivel de competencia del mercado, y de atributos personales, con especial destaque para el papel de la escolaridad. De la misma forma, los modelos con inspiración en Becker (1975) destacan que inversiones en capital humano, como la educación y el entrenamiento, son reflejadas en mayores rendimientos a lo largo del ciclo de vida laboral y mayores posibilidades de adaptación a nuevas tecnologías (De Hoyos, Popova y Rogers, 2016).

Dentro de la estructura de estos modelos, la condición de ni-ni implica en una situación en la cual el individuo no está acumulando capital humano, sea específico o general. El capital humano específico proviene de la experiencia en el lugar de trabajo, o del entrenamiento para realizar determinada tarea y que difícilmente puede ser desplazado para otra ocupación. De otro lado, el capital humano general es adquirido por la inversión en educación formal, financiada por recursos públicos o privados (Mincer y Jovanovic, 1979; Borjas, 2008). Ambos aumentan la productividad y, en consecuencia, los salarios de los trabajadores. Durante la situación de ni-ni, estas inversiones se encuentran prácticamente estancadas o inexistentes. Por lo tanto, cuando estos jóvenes decidieren, en un tiempo futuro, actuar en el mercado de trabajo, se espera de ellos un menor nivel de competitividad en comparación con trabajadores que no han pasado recientemente por esta condición, incluso después de controlado el nivel de escolaridad formal.

Este contexto es reflejado también en el proceso de contratación de las empresas que, cuando reciben diversas solicitudes de candidatos aceptables de empleo, realizan la contratación según un ranking. Las empresas contratan primero los candidatos que se encuentran a menos tiempo sin ejercer algún tipo de ocupación, puesto que presentan un más bajo deterioro de sus habilidades específicas. Es decir, se supone que el stock de capital humano acumulado por la experiencia, además del adquirido con la educación formal, puede ser perdido o tener su cualidad disminuida por la falta de uso, con efectos sobre la tasa de salida de la desocupación. Esta relación es observada, por ejemplo, en Blanchard y Diamond (1994), que argumentan, sobre todo en periodos de recesión económica, que los desocupados de largo plazo tienden a presentar probabilidades inferiores de contratación, imputando sobre ellos un estigma cada vez más significativo. 
De la misma formal, Pissarides (1992) muestra un mayor deterioro de las habilidades del trabajador que se encuentra a más tiempo sin una ocupación. Largas duraciones del desempleo desestimulan la oferta de nuevas plazas por las empresas, puesto que esto implica en menor calidad del conjunto de desempleados. La presencia de un choque negativo en el desempleo en un dado periodo disminuye el nivel de contrataciones, aumenta la duración del paro y contribuye a acentuar la pérdida de habilidades, principalmente las adquiridas por entrenamiento específico o clases profesionalizantes.

Además del capital humano, los jóvenes que se encuentran fuera del mercado de trabajo también presentan pérdida de capital social o de importantes conexiones sobre información de nuevas plazas de empleo. Montgomery (1991) observa que trabajadores que permanecen por más tiempo desocupados o fuera de la fuerza de trabajo pierden con más facilidad la capacidad de mantenerse actualizado acerca de nuevas plazas vacantes. De esta forma, jóvenes que pasan más tiempo en la condición de ni-ni se encuentran obsoletos para ser considerados en procesos de contratación, no acumulando capital humano y tampoco capital social, disminuyendo cada vez más las posibilidades de encontrar una ocupación adecuada.

Samoilenko y Carter (2015) muestran que quien permanece por mucho tiempo en la condición de no trabajar y no estudiar presenta peores resultados futuros en escolaridad media, menor empleabilidad y mayor inactividad. En Brasil, los estudios sobre las tasas de ni-ni tienen se intensificado a lo largo de los últimos años, usando sobre todo los microdatos de los censos demográficos y de la Pesquisa Nacional por Amostra de Domicílios (PNAD). Camarano (2006) utiliza los dados del censo de 1980 y 2000 para estimar modelos de probabilidad de observarse jóvenes entre 15 y 29 años que no trabajan y no estudian, tomando como variables explicativas características de los hogares y demográficas. Los resultados muestran que la educación tiene el impacto de reducir la probabilidad de ser ni-ni, que hogares con personas en esta situación tienden a presentar un menor nivel de renta y que esta renta es formada principalmente por programas sociales y donaciones.

Kovrona, Lyon y Rosati (2013) comparan las situaciones de ni-ni entre Brasil e Indonesia, argumentando que ambos los países pasaran por procesos de crecimiento económico combinado con elevaciones de las tasas de paro juvenil. Los datos brasileños muestran una disminución de la probabilidad combinada de estar fuera del mercado de trabajo y de los estudios entre las generaciones, siendo más expresivo entre las mujeres nascidas en la década del 1990 si comparado con las nascidas en los años 
del 1970. Cabanas, Komatsu y Menezes-Filho (2015) analizan la situación de los jóvenes con un modelo logit multinomial y variables explicativas que envuelven aspectos del hogar e individuales. Los resultados sugieren, por ejemplo, que aumentos en el nivel de renta del hogar reducen el coste de oportunidad de la inversión en educación de los jóvenes dependientes, favoreciendo la dedicación a los estudios y desestimulando el empleo remunerado orientado a formar la renta familiar. De la misma forma, aumentos en la escolaridad de los padres aumentan la probabilidad del joven asistir a una institución de enseñanza y disminuyen la posibilidad de que se encuentre trabajando. Estos resultados evidencian que el aumento reciente de la cantidad de jóvenes ni-ni es debido, entre otros factores, a aumentos reales en la renta de los hogares brasileños, puesto que desestimulan el empleo de los más jóvenes, pero estimulan menos que proporcionalmente la dedicación de estos mismos a los estudios.

Menezes-Filho, Cabanas y Komatsu (2013) analizan la duración de la permanencia de jóvenes con edad entre 17 e 22 años en la condición de ni-ni, utilizando matrices de transición con los datos de la Pesquisa Mensal de Emprego (PME) entre 2003 y 2011. Los resultados indican una disminución, a lo largo de los años, de la tasa de entrada en la condición de ni-ni para el subgrupo de mujeres, aunque tanto hombres como mujeres experimentaran aumentos significativos de duración media en la condición. También evidencian que los jóvenes con enseñanza fundamental incompleta poseen tiempo medio de permanencia y tasa de entrada considerablemente superiores a los demás grupos educacionales. La investigación sugiere que el tiempo medio de permanencia es relativamente pequeño, una vez que, después de un año, la proporción de jóvenes ni-ni que cambian de situación es mayor que la proporción de los que permanecen sin estudiar y trabajar. Según los autores, aumentos de la tasa de jóvenes ni-ni en los años recientes corresponden al aumento de la duración media en la condición, elevando la cantidad de personas que se encuentran desempleadas y no estudiando.

En síntesis, la literatura empírica muestra la importancia de considerar aspectos de los hogares y personales de los jóvenes para analizar su probabilidad de ser ni-ni. Sin embargo, la mayor parte de la literatura dedicada espacio para discutir la determinación estática de la tasa ni-ni en el país, a excepción de Menezes-Filho, Cabanas y Komatsu (2013) que abordan esta situación en un análisis dinámico para el Brasil metropolitano entre 2003 e 2011. El presente artículo dedica más espacio para analizar el tiempo en que los individuos jóvenes de 15 hasta 29 años permanecen en la condición de no trabajar, destacando sobre todo si el 
joven está utilizando este tiempo para dedicarse o no a una cualificación formal. También se investiga como que la actual situación en la cual el individuo se encuentra interfiere en su decisión de entrada en la condición de ni-ni, para captar relaciones de dependencia temporal. Adicionalmente, el análisis cubre un periodo más extenso, que permite observar los efectos de la pérdida de dinamismo de la economía brasileña en los años más recientes, después del registro de avance socioeconómico en la primera década del siglo XXI en el país.

\section{Procedimientos metodológicos}

La base de datos utilizada en esta investigación es la Pesquisa Mensal de Emprego (PME), un panel rotativo de hogares realizado por el Instituto Brasileiro de Geografía e Estatística (IBGE), entre los años de 2002 y $2015^{3}$ en seis regiones metropolitanas de Brasil (Recife, Salvador, Belo Horizonte, Rio de Janeiro, São Paulo y Porto Alegre). Son considerados jóvenes los individuos con edad entre 15 y 29 años, de acuerdo con la mayor parte de la literatura nacional, y definidos como nini aquellos que se encuentran fuera de la Población Económicamente Activa (PEA) y, al mismo tiempo, no frecuentando la escuela.

La denominación de panel rotativo significa que, cada mes, la muestra es gradualmente sustituida por un nuevo conjunto de hogares. Cada hogar participa de la encuesta por un periodo de cuatro meses consecutivos y, después, deja de ser investigado por ocho meses. Después de este periodo, vuelve a entrar en la encuesta por cuatro meses más y sale de forma definitiva de la muestra. Esta estructura permite que las condiciones de vida de los hogares y de sus residentes sean comparadas, por ejemplo, mes a mes o en el intervalo de un año. Es utilizada, por ejemplo, en Moreira y Queiroz (2017) para investigar la transición laboral de personas mayores de 50 años, en Nunes et al. (2016) para análisis de despidos y contrataciones, en Cacciamali, Lima y Tatei (2015) para estimar la duración del desempleo y en Menezes-Filho, Cabanas y Komatsu (2013) para análisis de los ni-ni. Para los objetivos del presente artículo, son comparadas las características de la inserción en el mercado de trabajo de los individuos en la primera $(t)$ y en la quinta $(t+1)$

\footnotetext{
${ }^{3}$ Pese a que la encuesta se encierre en febrero de 2016, se ha optado por considerar las estimativas hasta diciembre de 2015, para evitar posibles problemas a causa de componentes estacionales de los meses de enero y febrero.
} 
entrevistas llevadas a cabo en el hogar, separadas por un periodo de un año ${ }^{4}$.

Dos métodos de análisis son utilizados. El primero es un análisis univariado con base en el trabajo de Clark y Summers (1990), que asume que el comportamiento del individuo, según sus características personales, puede ser representado por una matriz de probabilidad de transición entre cuatro estados o situaciones en el mercado de trabajo: PEA y estudiando; PEA y no estudiando, Fuera de la PEA y estudiando y; Fuera de la PEA y sin estudiar (los ni-ni). La dinámica de la movilidad entre estos cuatro estados es definida por una matriz de Markov, que define la probabilidad del individuo $i$ estar en la condición $k$ en el periodo $t+1$, una vez que se encuentra en la condición $l$ en el periodo $t$. La representación de la matriz de transición es definida por:

$$
p^{i}=\left[\begin{array}{llll}
p_{11} & p_{12} & p_{13} & p_{11} \\
p_{21} & p_{22} & p_{23} & p_{24} \\
p_{31} & p_{32} & p_{33} & p_{34} \\
p_{41} & p_{42} & p_{43} & p_{44}
\end{array}\right]
$$

en el cual $P^{i} l_{l, k}$ representa la probabilidad del individuo $i$ estar en la situación $k$ (representada por el numeral " 1 " para PEA y estudiando; " 2 " para PEA y no estudiando; " 3 " para Fuera de la PEA y estudiando y; " 4 " para Fuera de la PEA y sin estudiar) en el período $t+1$, condicionado al hecho de encontrarse en la condición $l$ en el período $t$. Por la matriz $p^{i}$ se calcula la proporción de tiempo dedicado en cada condición del mercado de trabajo por cada individuo $i$. Considerando $\pi_{j}^{i}$ como la fracción de tiempo en que el individuo $i$ permanece en la situación $j$, es posible obtener:

$$
\pi_{j}^{i}=\left[\begin{array}{l}
\pi_{1}^{i} \\
\pi_{2}^{i} \\
\pi_{3}^{i} \\
\pi_{4}^{i}
\end{array}\right]
$$

Dado que $\pi_{j}^{i}$ no es una variable directamente observable, se considera la hipótesis de que las transiciones entre las situaciones son tratadas como un proceso de Markov, en lo cual el desarrollo futuro del proceso, puesto que el individuo se encuentra en una situación, depende

\footnotetext{
${ }^{4}$ Se utilizó el algoritmo propuesto en Ribas y Soares (2008) para minimizar problemas de pérdida de información entre cada entrevista.
} 
únicamente de esta misma situación y no de cómo se llegó en la misma. Por este sistema, por lo tanto, la relación entre $\pi_{t}^{i} \mathrm{y} \pi_{t-1}^{i}$ puede ser escrita como:

$$
\pi_{t}^{i}=p_{i}^{\prime} \pi_{t-1}^{i}
$$

Usando el Teorema Básico de las Cadenas de Markov, que postula que cualquiera sistema caracterizado por una matriz de transición con estas características llega a un estado estacionario que es independiente de las condiciones iniciales, con probabilidades de estar en cada estado distribuida de forma uniforme entre los periodos, en este estado estacionario será válida la relación $\pi_{t}^{i}=\pi_{t-1}^{i}$, de modo que $\pi_{t}^{i}=p_{i}^{\prime} \pi_{t}^{i}$. Utilizando la condición de estado estacionario y el hecho de que cualquier de las probabilidades de transición entre las cuatro condiciones son independientes del tiempo en el cual cada individuo permanece en una situación particular:

$$
\grave{p_{i}} \pi_{t}^{i}=\pi_{t}^{i} \rightarrow\left[\begin{array}{llll}
p_{11}^{i} & p_{21}^{i} & p_{31}^{i} & p_{41}^{i} \\
p_{12}^{i} & p_{22}^{i} & p_{32}^{i} & p_{42}^{i} \\
p_{13}^{i} & p_{23}^{i} & p_{33}^{i} & p_{43}^{i} \\
p_{14}^{i} & p_{24}^{i} & p_{34}^{i} & p_{44}^{i}
\end{array}\right]\left[\begin{array}{c}
\pi_{1}^{i} \\
\pi_{1}^{i} \\
\pi_{1}^{i} \\
\pi_{1}^{i}
\end{array}\right]=\left[\begin{array}{c}
\pi_{1}^{i} \\
\pi_{2}^{i} \\
\pi_{3}^{i} \\
\pi_{4}^{i}
\end{array}\right]
$$

Y, la proporción de tiempo en cada estado puede ser representada como:

$$
\begin{aligned}
& \Rightarrow p_{11}^{i} \pi_{1}^{i}+p_{21}^{i} \pi_{2}^{i}+p_{31}^{i} \pi_{3}^{i}+p_{41}^{i} \pi_{4}^{i}=\pi_{1}^{i} \\
& \Rightarrow p_{12}^{i} \pi_{1}^{i}+p_{22}^{i} \pi_{2}^{i}+p_{32}^{i} \pi_{3}^{i}+p_{42}^{i} \pi_{4}^{i}=\pi_{2}^{i} \\
& \Rightarrow p_{13}^{i} \pi_{1}^{i}+p_{23}^{i} \pi_{2}^{i}+p_{33}^{i} \pi_{3}^{i}+p_{43}^{i} \pi_{4}^{i}=\pi_{3}^{i} \\
& \Rightarrow p_{14}^{i} \pi_{1}^{i}+p_{24}^{i} \pi_{2}^{i}+p_{34}^{i} \pi_{3}^{i}+p_{44}^{i} \pi_{4}^{i}=\pi_{4}^{i}
\end{aligned}
$$

Cualquiera de las ecuaciones de este sistema linear es dependiente una de las otras. Sin embargo, se puede utilizar la propiedad de que $\pi_{1}^{i}+\pi_{2}^{i}+\pi_{3}^{i}+\pi_{4}^{i}=1$, substituir en cualquier una de las ecuaciones para resolver el sistema y obtener las proporciones de tiempo deseadas. Estas proporciones, a su turno, son consideradas como estimaciones del tiempo que el individuo permanece en cada una de las cuatro condiciones del mercado de trabajo analizadas a lo largo de su ciclo de vida.

La segunda metodología toma por base un análisis econométrico multivariado, con la estimación de un modelo logit multinomial, como presentado en Wooldridge (2007). Con este modelo es posible entender como los cambios en el conjunto de variables relativas a atributos 
personales y relacionados con la coyuntura económica afectan la probabilidad de un individuo participar o no de la población económicamente activa (PEA) y su decisión de frecuentar o no la escuela.

El modelo es estimado para determinar la probabilidad de que, en el momento de la quinta entrevista $(t+1)$, el joven se encuentre en una de cuatro situaciones distintas: en la PEA y estudiando; en la PEA pero sin estudiar; fuera de la PEA y sin estudiar (los ni-ni, o la categoría de principal interés); y fuera de la PEA y estudiando, siendo esta la categoría de referencia para la estimación. Como variables explicativas, se utiliza una dummy para el color de la piel (blancos y negros y pardos), dummies para la condición en el hogar (cónyuge, hijo; otros miembros), años de educación formal, la edad, dummies anuales para captar efectos macroeconómicos, y dummies para las regiones metropolitanas. Además, son utilizadas dummies para identificar la condición en la cual el individuo se encontraba en el periodo $t$ (es decir, la primera entrevista, realizada un año antes), para captar dependencia temporal de las condiciones del mercado de trabajo.

Acerca de este modelo, debe ser considerado el riesgo de endogeneidad, a causa del uso de variables explicativas que reflejan la situación de los mismos individuos un año antes. Tomando como referencia los trabajo de Wooldridge (2014) y Geraci, Fabbri y Monfardini (2018), se realizó un contrate para la hipótesis de endogeneidad con un método de variables instrumentales, estimando un logit multinomial para la condición anterior en función de las variables fijas en el tiempo (raza, dummies anuales y regionales). A continuación, los errores estándar son extraídos y utilizados como variables explicativas adicionales en la ecuación principal. En el anexo se muestra, sin embargo, que los coeficientes estimados son, en su mayoría, no significativos, lo que lleva a concluir por la no endogeneidad de las variables.

\section{Resultados}

Para el análisis, los jóvenes son divididos en tres grupos etarios, de 15 hasta 17 años; de 18 hasta 24 años y de 25 hasta 29 años, para realizar comparaciones de la decisión entre trabajo, escuela e inactividad en puntos distintos del ciclo de vida (De Hoyos, Popova y Rogers, 2016). Como ha sido definido anteriormente, los ni-ni son los jóvenes que se encuentran simultáneamente fuera de la fuerza de trabajo y fuera de la escuela. Estos, corresponden a cerca de un $13 \%$ de la muestra, siendo de mayor magnitud entre las mujeres (18\%) que entre los varones (8\%) en 
la media del periodo analizado. El cuadro 1 exhibe el tamaño de las submuestras utilizadas. Los mayores problemas de tamaño muestral son de varones y mujeres más jóvenes en la PEA sin estudiar y varones fuera de la PEA, pero en la escuela. Los grupos de ni-ni, sin embargo, presentan muestras con mayor número de observaciones y parecen contemplar las propiedades asintóticas de las metodologías utilizadas.

Cuadro 1. Número de observaciones total y de las submuestras total del periodo. Fuente: elaboración propia con datos de la PME.

\begin{tabular}{lrrrrc}
\hline Mujeres & PEA y Estudia & PEA y no Estudia & Ni-Ni & No PEA y Estudia & Total \\
\hline 15/17 años & 2.852 & 669 & 1.257 & 16.411 & 21.189 \\
$18 / 24$ años & 8.892 & 19.014 & 9.294 & 8.420 & 45.620 \\
$25 / 29$ años & 3.304 & 19.122 & 7.792 & 1.250 & 31.468 \\
Total & 15.048 & 38.805 & 18.343 & 26.081 & 98.277 \\
\hline Varones & PEA y Estudia & PEA y no Estudia & Ni-Ni & No PEA y Estudia & Total \\
\hline 15/17 años & 3.541 & 888 & 1.077 & 15.669 & 21.175 \\
$18 / 24$ años & 9.556 & 23.114 & 5.054 & 7.059 & 44.783 \\
$25 / 29$ años & 3.224 & 21.584 & 2.140 & 671 & 27.619 \\
Total & 16.321 & 45.586 & 8.271 & 23.399 & 93.577 \\
\hline
\end{tabular}

Para seguir la evolución del comportamiento de los jóvenes en relación al mercado de trabajo brasileño, el gráfico 1 exhibe la tasa de desocupación, segmentada según grupos de edad y de sexo, de la población económicamente activa dentro del periodo de análisis. A partir de la mitad de 2003, el mercado de trabajo responde de forma positiva a un movimiento de expansión de la economía brasileña, generando una tendencia continuada de disminución del desempleo hasta 2012. Después de esto, sin embargo, se observa una inversión de esta tendencia de forma más acentuada entre los jóvenes de 15 hasta 17 años y para el grupo etario de 18 hasta 24 años, principalmente para las mujeres de la muestra.

Cuando se analiza la tasa de inactividad, con el gráfico 2, se observa un aumento de la inactividad para los grupos más jóvenes, entre 15 y 24 años, a partir de 2012, mientras los demás grupos presentan una tasa relativamente constante a lo largo de todo el periodo, siendo más expresivo para el sexo femenino. Llama la atención la forma ascendente 
de la tasa de inactividad, después de 2012, para el grupo de jóvenes adultos entre 18 y 24 años comparada a los grupos de mayor edad, tanto para jóvenes del sexo masculino como para las mujeres. El aumento de la tasa de desocupación y de inactividad entre los grupos más jóvenes muestra la importancia de analizar con más detalles como ocurre tanto la transición de esos grupos entre los estados del mercado de trabajo como también los determinantes de la entrada y la permanencia en cada una de estas situaciones.

Gráfico 1. Tasa de desocupación según grupos de edad y de sexo - Brasil metropolitano (2002-2015). Fuente: elaboración propia con los datos de la PME.

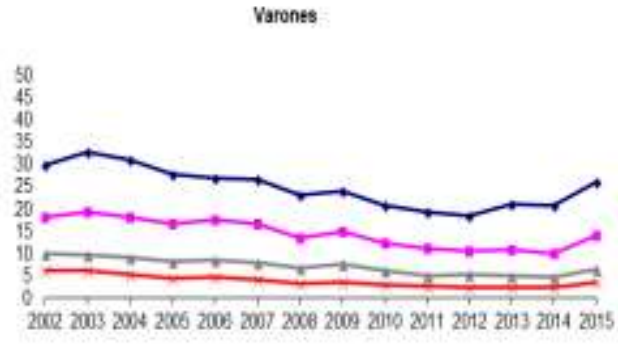

$\rightarrow-10-4 t+18-24+28-20+10-56$

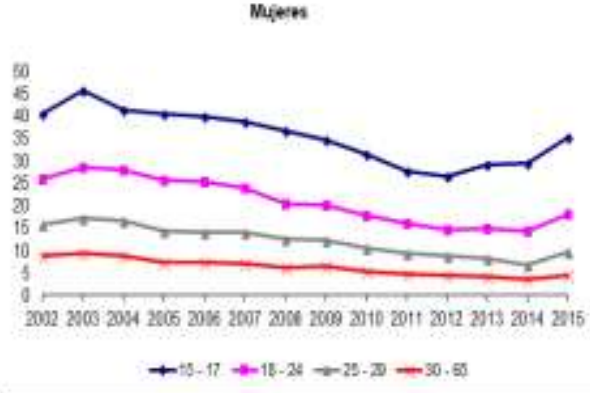

$+18-17+15 \cdot 24+28 \cdot 20 \div-30 \cdot 65$

Gráfico 2. Tasa de inactividad según grupos de edad y de sexo Brasil metropolitano (2002-2015). Fuente: elaboración propia con los datos de la PME.
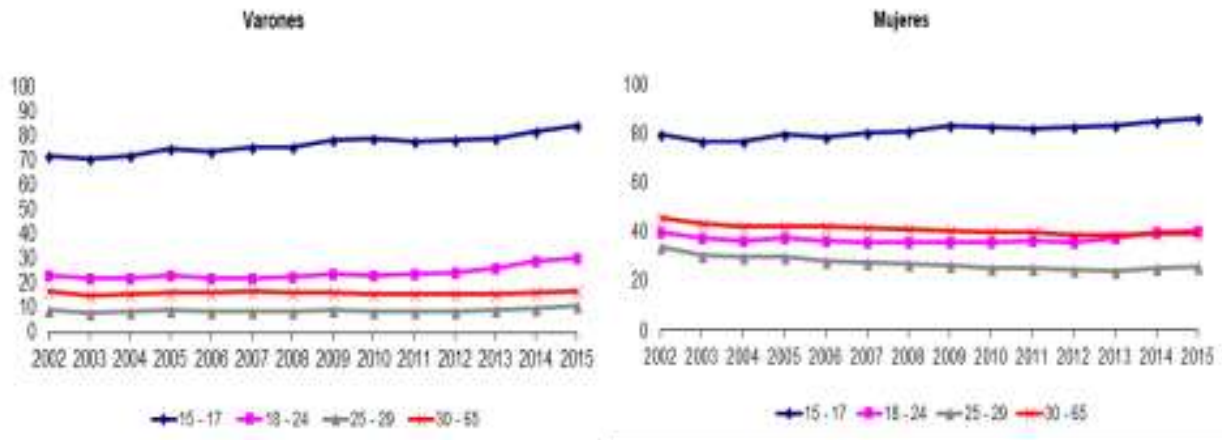
Para investigar la permanencia de los jóvenes en la PEA o en la inactividad, considerando la frecuencia o no a la escuela, son estimadas las fracciones de tiempo en cada estado del mercado de trabajo a partir de las matrices de transición de Markov. Los gráficos 3 y 4 muestran el resultado para la estimación del porcentaje medio de tiempo, en estado estacionario, que los jóvenes se encuentran en la inactividad estudiando o como inactivos sin estudiar respectivamente, obtenidas por la probabilidad de estar en cada estado en $t+1$, condicionado al hecho de estar en un determinado estado en $t$.

El aumento de la inactividad observada para los grupos etarios más jóvenes sería un resultado benéfico, si estos grupos estuviesen postergando la entrada en el mercado de trabajo para dedicarse exclusivamente a los estudios y, por lo tanto, a la adquisición de capital humano general. Este es un escenario positivo para los jóvenes entre 15 y 17 años como muestra el gráfico 3. Entre estos individuos de menor edad, parece existir una mayor influencia de la presión familiar para mantenerse en alguna actividad productiva o educacional, conforme argumenta el modelo de De Hoyos, Popova y Rogers (2016). Sin embargo, para los jóvenes con edad entre 18 y 24 años y entre 25 y 29, los resultados muestran un aumento más expresivo en la proporción de tiempo sin estudiar y fuera del mercado de trabajo, sobre todo entre los del primero grupo de ambos los sexos (gráfico 4). Los resultados, por lo tanto, sugieren que el aumento en el tiempo dedicado en la inactividad por estos grupos no está relacionado a una mayor acumulación de capital humano.

Las matrices de transición representan un análisis de largo plazo, o de estado estacionario, del comportamiento de los individuos. Los resultados muestran el porcentaje de tiempo medio que los grupos de jóvenes permanecen en cada situación del mercado de trabajo a lo largo del ciclo de vida considerado. De esta forma, aumentos en la fracción de tiempo dedicada en la condición de ni-ni significa, a largo plazo, por lo menos una generación de trabajadores con riesgo de una caída brusca de su productividad.

Para un análisis de corto plazo, sin embargo, es posible observar como las transiciones entre los estados del mercado de trabajo y, en particular, para la condición de ni-ni, depende de los atributos personales, de factores agregados y también de la posición anterior de los individuos. Este análisis es realizado con un modelo logit multinomial, con sus resultados exhibidos en los cuadros 2 y 3 , y que utiliza como variable explicada una cualitativa que separa los individuos de la muestra en cuatro situaciones del mercado de trabajo. 
Gráfico 3. Porcentaje de tiempo en estado estacionario en la inactividad y estudiando, según grupos de edad y de sexo - Brasil metropolitano (2003-2015). Fuente: elaboración propia con los datos de la PME.
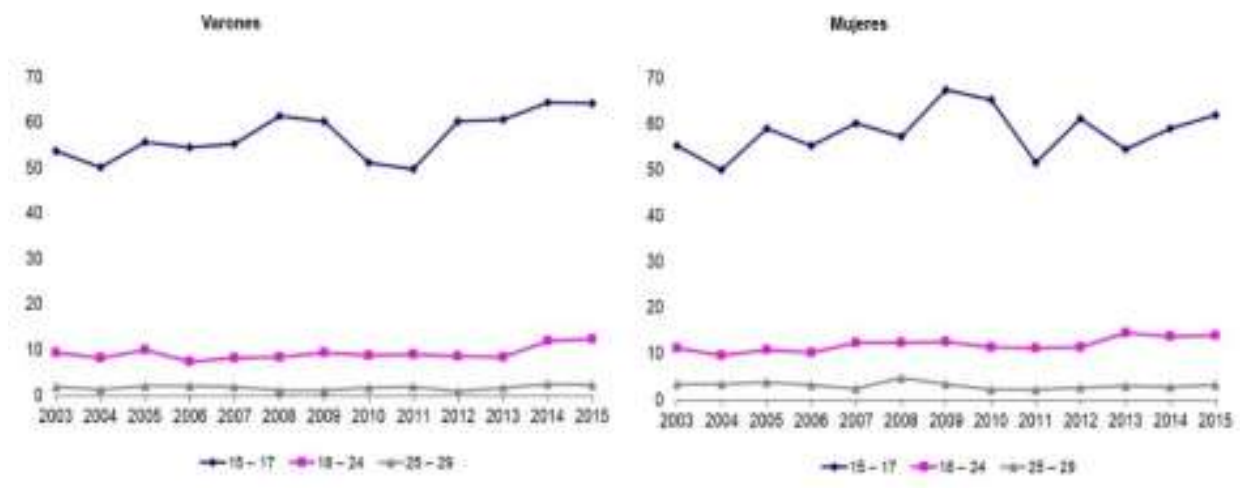

Gráfico 4. Porcentaje de tiempo en estado estacionario en la inactividad y sin estudiar, según grupos de edad y de sexo Brasil metropolitano (2003-2015). Fuente: elaboración propia con los datos de la PME.


Los resultados son exhibidos utilizando los efectos marginales, que captan el cambio en la probabilidad de cada status o situación de actividad cuando uno de los factores explicativos sufre una variación. Como se tratan de variables explicativas binarias en su mayoría, los efectos representan la diferencia de probabilidad entre los diversos grupos considerados y las categorías de referencia omitidas. Por 
simplificación, los cuadros omiten los efectos marginales para la situación de estar en la PEA y estudiando.

En líneas generales, se constatan diferencias significativas en los determinantes de entrada en la inactividad de los jóvenes que estudian y los que no estudian. Jóvenes negros y pardos, con menor escolaridad, residentes en las regiones metropolitanas de Salvador y de Rio de Janeiro, presentan mayores probabilidades de ser parte de los inactivos, sobre todo como ni-ni, es decir, fuera de la escuela. Las dummies anuales evidencian el aumento de la probabilidad de entrar en la condición de nini a lo largo de los años, con mayor expresión en el periodo más reciente, sobre todo para los jóvenes con edad entre 25 y 29 años.

A partir de 2014, la economía brasileña presenta señales de recesión económica, intensificada por una crisis política, que detiene el avance logrado durante el inicio del siglo XXI en el país. El bajo dinamismo de la economía, con deterioro del producto interno bruto y de la tasa de ocupación, fue sentido principalmente por los más vulnerables, como los más jóvenes, como muestran los resultados encontrados por las binarias temporales, sobre todo para aquellos con edad entre 25 y 29 años. Estos resultados confirman los hallazgos de la literatura empírica previa para el Brasil, como en Camarano (2006) y Menezes-Filho, Cabanas y Komatsu (2013).

Los modelos estimados también muestran una significativa dependencia temporal de la situación anterior de los jóvenes, especialmente para aquellos que no son parte de la PEA y que no estaban estudiando en el periodo anterior, dificultando aún más la salida de esta condición. Esto contribuye a una mayor vulnerabilidad de los jóvenes en la condición de ni-ni, y significa, según la literatura, que existe un mayor desgaste del capital humano previamente acumulado por la falta de uso y de entrenamiento continuo. Quienes que son parte de la PEA, pero sin estudiar en el periodo anterior, también presentan una probabilidad más alta de entrar en la categoría ni-ni al periodo siguiente, para el caso de los individuos más jóvenes de la muestra. Es decir, incluso la condición de los más jóvenes que se encuentran trabajando, pero que se encuentran alejados del sistema educacional, puede facilitar la entrada en la situación de ni-ni. 
Cuadro 2. Efectos marginales estimados para los varones en $t+1$ - Brasil Metropolitano (2002-2015). Fuente: elaboración propia con los datos de la PME.

\begin{tabular}{|c|c|c|c|c|c|c|c|c|c|}
\hline & \multicolumn{3}{|c|}{ Fuera de la PEA y Estudia } & \multicolumn{3}{|c|}{ PEA y no Estudia } & \multicolumn{3}{|c|}{$\mathrm{Ni}-\mathrm{Ni}$} \\
\hline & $\begin{array}{l}15 / 17 \\
\text { años }\end{array}$ & $\begin{array}{l}18 / 24 \\
\text { años }\end{array}$ & $\begin{array}{l}25 / 29 \\
\text { años }\end{array}$ & $\begin{array}{l}15 / 17 \\
\text { años }\end{array}$ & $\begin{array}{l}18 / 24 \\
\text { años }\end{array}$ & $\begin{array}{l}25 / 29 \\
\text { años }\end{array}$ & $\begin{array}{l}15 / 17 \\
\text { años }\end{array}$ & $\begin{array}{l}18 / 24 \\
\text { años }\end{array}$ & $\begin{array}{l}25 / 29 \\
\text { años }\end{array}$ \\
\hline PEA y estudia en $t$ & $-0,41^{*}$ & $-0,10^{*}$ & $-0,01^{*}$ & $0,04 *$ & $0,11^{*}$ & $0,04^{*}$ & 0,00 & $-0,11^{*}$ & $-0,05^{*}$ \\
\hline PEA y no estudia en $t$ & $-0,64^{*}$ & $-0,31^{*}$ & $-0,21^{*}$ & $0,41^{*}$ & $0,59 *$ & $0,58^{*}$ & $0,09 *$ & $-0,06^{*}$ & $-0,10^{*}$ \\
\hline Ni-Ni en $t$ & $-0,33^{*}$ & $-0,08^{*}$ & $-0,01^{*}$ & $0,09 *$ & $0,22^{*}$ & $0,07 *$ & $0,27^{*}$ & $0,05^{*}$ & 0,01 \\
\hline Blancos & $0,05^{*}$ & $0,05^{*}$ & $0,00 *$ & $-0,01^{*}$ & $-0,10^{*}$ & $-0,01^{*}$ & $-0,01^{*}$ & $-0,01^{*}$ & 0,00 \\
\hline Cónyuge & 0,06 & $-0,01$ & $-0,00$ & $-0,00$ & $-0,02$ & 0,00 & $-0,01$ & 0,04 & 0,01 \\
\hline Hijo & 0,12 & $0,04^{*}$ & $0,01^{*}$ & $-0,03$ & $-0,19^{*}$ & $-0,07^{*}$ & 0,01 & $0,07 *$ & $0,04^{*}$ \\
\hline Otros de la familia & 0,07 & $0,04^{*}$ & $0,01^{* *}$ & $0,01^{* * *}$ & $-0,18^{*}$ & $-0,06^{*}$ & 0,01 & $0,10^{*}$ & $0,04^{*}$ \\
\hline 4/7 años de estudio & 0,01 & $-0,02^{*}$ & $-0,00$ & $-0,00$ & $0,07^{*}$ & $0,06^{*}$ & $-0,02 *$ & $-0,08^{*}$ & $-0,04^{*}$ \\
\hline 8/10 años de estudio & $0,07 *$ & 0,01 & $-0,00$ & $-0,02 *$ & $-0,04$ & $0,04^{*}$ & $-0,09 *$ & $-0,13^{*}$ & $-0,05^{*}$ \\
\hline $\begin{array}{l}11 \text { o más años de } \\
\text { estudio }\end{array}$ & $-0,16^{*}$ & $-0,06^{*}$ & $-0,00$ & $0,13^{*}$ & $0,18^{*}$ & $0,12^{*}$ & $0,09^{*}$ & $-0,16^{*}$ & $-0,13^{*}$ \\
\hline Edad & $-0,08^{*}$ & $-0,01^{*}$ & $-0,00^{*}$ & $0,01^{*}$ & $0,01^{*}$ & $0,01^{*}$ & $0,01^{*}$ & $-0,01^{*}$ & $-0,00$ \\
\hline Recife & $0,09^{*}$ & $0,06^{*}$ & $0,01^{*}$ & $-0,01^{*}$ & $-0,12^{*}$ & $-0,08^{*}$ & $0,01^{* * *}$ & $0,06^{*}$ & $0,05^{*}$ \\
\hline Salvador & $0,06^{*}$ & $0,09 *$ & $0,01^{*}$ & $-0,01^{*}$ & $-0,16^{*}$ & $-0,06^{*}$ & $-0,01^{*}$ & $0,02 * *$ & $0,03^{*}$ \\
\hline Belo Horizonte & $-0,00$ & $0,02 *$ & $0,01^{*}$ & $-0,00$ & $-0,06^{*}$ & $-0,05^{*}$ & 0,00 & $0,02 *$ & $0,02^{*}$ \\
\hline Rio de Janeiro & $0,12^{*}$ & $0,07 *$ & $0,01^{*}$ & $-0,01^{*}$ & $-0,06^{*}$ & $-0,01 * *$ & $-0,01^{*}$ & $0,01 * *$ & 0,00 \\
\hline Porto Alegre & $-0,03 * *$ & $-0,00$ & $0,01^{*}$ & $0,01^{*}$ & $-0,01$ & $-0,05^{*}$ & $0,02^{*}$ & $0,01^{* * *}$ & $0,02^{*}$ \\
\hline 2004 & 0,01 & $-0,01$ & $-0,00$ & $-0,00$ & 0,02 & $0,02^{* *}$ & 0,00 & 0,01 & 0,01 \\
\hline 2005 & 0,02 & 0,00 & $-0,00$ & $-0,00$ & 0,02 & 0,01 & 0,00 & 0,02 & 0,01 \\
\hline 2006 & 0,02 & $-0,01$ & $-0,00$ & 0,00 & $0,04^{* *}$ & 0,01 & 0,00 & $-0,01$ & $-0,00$ \\
\hline 2007 & $0,02^{* * *}$ & $-0,00$ & $-0,00$ & $-0,00$ & 0,01 & $0,02^{* *}$ & 0,00 & $0,02^{* * *}$ & $-0,00$ \\
\hline 2008 & $0,05^{*}$ & 0,00 & $-0,00$ & $-0,00 * *$ & $-0,01$ & 0,01 & $-0,00$ & $0,04^{*}$ & 0,00 \\
\hline 2009 & $0,05^{*}$ & 0,01 & $0,00^{* * * *}$ & $-0,01^{*}$ & $-0,01$ & 0,01 & 0,00 & $0,04^{*}$ & 0,00 \\
\hline 2010 & $0,05^{*}$ & 0,00 & $-0,00$ & $-0,00$ & 0,00 & 0,00 & $0,01 * * *$ & $0,03^{* *}$ & 0,01 \\
\hline 2011 & $0,04^{*}$ & 0,01 & $-0,00$ & 0,00 & $-0,01$ & 0,01 & 0,01 & $0,03 * *$ & 0,01 \\
\hline 2012 & $0,05^{*}$ & 0,01 & $-0,00 * *$ & $-0,00$ & $0,04^{* *}$ & 0,02 & 0,01 & $0,05^{*}$ & 0,01 \\
\hline 2013 & $0,05^{*}$ & 0,00 & $-0,00$ & $-0,00$ & $-0,01$ & $-0,00$ & 0,01 & $0,06^{*}$ & $0,02^{* * *}$ \\
\hline 2014 & $0,07 *$ & $0,03^{*}$ & 0,00 & $-0,00$ & $-0,05^{*}$ & 0,00 & $0,02^{* *}$ & $0,08^{*}$ & $0,02 * *$ \\
\hline 2015 & $0,08^{*}$ & $0,03^{*}$ & 0,00 & $-0,00$ & $-0,05^{*}$ & $-0,01$ & 0,01 & $0,07 *$ & $0,03^{*}$ \\
\hline Pseudo - $\mathrm{R}^{2}$ & 0,2660 & 0,2751 & 0,3051 & & & & & & \\
\hline Número de obs. & 20963 & 44429 & 27397 & & & & & & \\
\hline $\mathrm{Chi}^{2}$ & 8844,1 & 29109,8 & 11530,7 & & & & & & \\
\hline Prob $>\mathrm{Chi}^{2}$ & 0,00 & 0,00 & 0,00 & & & & & & \\
\hline
\end{tabular}




\section{Cuadro 3. Efectos marginales estimados para las mujeres en $t+1$ - Brasil Metropolitano (2002-2015)}

\begin{tabular}{|c|c|c|c|c|c|c|c|c|c|}
\hline & \multicolumn{3}{|c|}{ Fuera de la PEA y Estudia } & \multicolumn{3}{|c|}{ PEA y no Estudia } & \multicolumn{3}{|c|}{$\mathrm{Ni}-\mathrm{Ni}$} \\
\hline & $15 / 17$ & $18 / 24$ & $25 / 29$ & $15 / 17$ & $18 / 24$ & $25 / 29$ & $15 / 17$ & $18 / 24$ & $25 / 29$ \\
\hline & años & años & años & años & años & años & años & años & años \\
\hline PEA y estudia en $t$ & $-0,34^{*}$ & $-0,12^{*}$ & $-0,02^{*}$ & $0,03^{*}$ & $0,14^{*}$ & $0,14^{*}$ & 0,00 & $-0,17^{*}$ & $-0,18^{*}$ \\
\hline $\begin{array}{l}\text { PEA y no estudia en } \\
t\end{array}$ & $-0,62^{*}$ & $-0,29 *$ & $-0,21^{*}$ & $0,33^{*}$ & $0,53^{*}$ & $0,53^{*}$ & $0,16^{*}$ & $-0,09 *$ & $-0,20 *$ \\
\hline $\mathrm{Ni}-\mathrm{Ni}$ en $t$ & $-0,41^{*}$ & $-0,14^{*}$ & $-0,04^{*}$ & $0,11^{*}$ & $0,18^{*}$ & 0,02 & $0,34^{*}$ & $0,14^{*}$ & $0,12^{*}$ \\
\hline Blancos & $0,03^{*}$ & $0,06^{*}$ & $0,01^{*}$ & $-0,00^{*}$ & $-0,09^{*}$ & $-0,00$ & $-0,01^{*}$ & $-0,04^{*}$ & $-0,01$ \\
\hline Cónyuge & $-0,04$ & $-0,01$ & 0,00 & 0,02 & $-0,06^{*}$ & $-0,10^{*}$ & 0,06 & $0,12^{*}$ & $0,10^{*}$ \\
\hline Hijo & 0,16 & $0,08^{*}$ & $0,01^{*}$ & $-0,01$ & $-0,09 *$ & $-0,00$ & $-0,08 * * *$ & $-0,08^{*}$ & $-0,03^{*}$ \\
\hline Otros de la familia & $0,08^{* * *}$ & $0,09 *$ & $0,01^{* *}$ & $-0,01^{* *}$ & $-0,12^{*}$ & $-0,05^{*}$ & $-0,02^{*}$ & $-0,05^{*}$ & 0,01 \\
\hline $4 / 7$ años de estudio & $0,05^{*}$ & $-0,00$ & $-0,01 * * *$ & 0,00 & $0,06 * * *$ & $0,05^{* *}$ & $-0,02^{*}$ & $-0,09^{*}$ & $-0,04^{*}$ \\
\hline 8/10 años de estudio & $0,09 *$ & 0,02 & 0,00 & $-0,01$ & $-0,04$ & $0,06^{*}$ & $-0,10^{*}$ & $-0,16^{*}$ & $-0,08^{*}$ \\
\hline $\begin{array}{l}11 \text { o más años de } \\
\text { estudio }\end{array}$ & $-0,32^{*}$ & $-0,09^{*}$ & $-0,02^{*}$ & $0,23^{*}$ & $0,25^{*}$ & $0,18^{*}$ & $0,11^{*}$ & $-0,21^{*}$ & $-0,17^{*}$ \\
\hline Edad & $-0,08^{*}$ & $-0,01^{*}$ & $-0,00$ & $0,01^{*}$ & $0,01^{*}$ & $0,01^{*}$ & $0,01^{*}$ & $-0,01^{*}$ & $-0,00 * *$ \\
\hline Recife & $0,07 *$ & $0,08^{*}$ & $0,02 *$ & $-0,01^{*}$ & $-0,15^{*}$ & $-0,13^{*}$ & $-0,00$ & $0,06^{*}$ & $0,09 *$ \\
\hline Salvador & $0,05^{*}$ & $0,09 *$ & $0,02^{*}$ & $-0,01^{*}$ & $-0,15^{*}$ & $-0,07^{*}$ & $-0,01$ & $0,02 * * *$ & $0,03^{* *}$ \\
\hline Belo Horizonte & 0,01 & $0,03^{*}$ & $0,01 * *$ & $-0,00 * *$ & $-0,07 *$ & $-0,03^{*}$ & 0,00 & 0,01 & 0,01 \\
\hline Rio de Janeiro & $0,10^{*}$ & $0,09 *$ & $0,01^{*}$ & $-0,01^{*}$ & $-0,10^{*}$ & $-0,04^{*}$ & $-0,01^{*}$ & $0,03^{*}$ & $0,03^{*}$ \\
\hline Porto Alegre & $-0,02 * *$ & $0,01 * *$ & $0,01 * *$ & 0,00 & $-0,06^{*}$ & $-0,03^{*}$ & $0,01 * *$ & 0,01 & 0,01 \\
\hline 2004 & 0,02 & $-0,02^{*}$ & 0,00 & $-0,00$ & 0,02 & $-0,00$ & $-0,01$ & 0,02 & $-0,01$ \\
\hline 2005 & $0,03 *$ & $-0,00$ & 0,00 & $-0,01^{*}$ & $-0,03$ & $-0,01$ & $-0,01^{*}$ & $0,04^{*}$ & 0,01 \\
\hline 2006 & $0,02^{* *}$ & $-0,01$ & $-0,00$ & $-0,00^{*}$ & 0,01 & 0,00 & $-0,01$ & $0,02^{* * *}$ & $-0,00$ \\
\hline 2007 & $0,04^{*}$ & 0,01 & $-0,00$ & $-0,00^{*}$ & $-0,02$ & $-0,00$ & $-0,01^{*}$ & $0,03^{* *}$ & 0,00 \\
\hline 2008 & $0,04^{*}$ & 0,01 & 0,01 & $-0,00^{*}$ & $-0,02$ & $-0,00$ & $-0,01 * * *$ & $0,03^{* *}$ & $-0,00$ \\
\hline 2009 & $0,06^{*}$ & 0,01 & 0,00 & $-0,00^{*}$ & $-0,04^{* *}$ & $-0,01$ & $-0,00$ & $0,04^{* *}$ & 0,00 \\
\hline 2010 & $0,05^{*}$ & 0,01 & $-0,00$ & $-0,00^{*}$ & $-0,02$ & 0,00 & $-0,00$ & $0,03^{* *}$ & 0,00 \\
\hline 2011 & $0,03^{* *}$ & 0,01 & $-0,00$ & $-0,00^{*}$ & $-0,06^{*}$ & $-0,00$ & $-0,00$ & $0,05^{*}$ & 0,00 \\
\hline 2012 & $0,05^{*}$ & 0,01 & $-0,00$ & $-0,00^{*}$ & $-0,04 * *$ & $-0,01$ & $-0,01$ & $0,03 * *$ & 0,01 \\
\hline 2013 & $0,05^{*}$ & $0,02^{* * *}$ & 0,00 & $-0,01^{*}$ & $-0,05^{*}$ & 0,01 & $-0,00$ & $0,05^{*}$ & 0,00 \\
\hline 2014 & $0,07 *$ & $0,02^{* *}$ & 0,00 & $-0,00^{*}$ & $-0,06^{*}$ & $-0,01$ & $-0,00$ & $0,06^{*}$ & 0,01 \\
\hline 2015 & $0,08^{*}$ & $0,03^{*}$ & 0,00 & $-0,00^{*}$ & $-0,08^{*}$ & $-0,02$ & $-0,00$ & $0,07^{*}$ & 0,02 \\
\hline Pseudo - $\mathrm{R}^{2}$ & 0,2972 & 0,2694 & 0,3096 & & & & & & \\
\hline Número de obs. & 21028 & 45298 & 31222 & & & & & & \\
\hline $\mathrm{Chi}^{2}$ & 9317,5 & 31930,9 & 18946,5 & & & & & & \\
\hline Prob $>\mathrm{Chi}^{2}$ & 0,00 & 0,00 & 0,00 & & & & & & \\
\hline
\end{tabular}

Fuente: elaboración propia con los datos de la PME. ${ }^{* * *} \mathrm{p}<0.10,{ }^{* *} \mathrm{p}<0.05,{ }^{*} \mathrm{p}<0.01$. Output completo con los autores.

En conjunto, las matrices de transición y los modelos de probabilidad evidencian que existe una tendencia de no utilización del tiempo de inactividad para la búsqueda de cualificación entre los jóvenes del Brasil metropolitano. El aumento observado en las tasas de 
desocupación y de inactividad entre los jóvenes parece no estar siendo seguido por un aumento de la cualificación de la mano de obra, principalmente en el periodo más reciente. Las implicancias de estos resultados son que, si en momentos de crisis económica, con efectos sobre la capacidad del mercado de trabajo de generar o mantener puestos de empleo, si la mano de obra más joven no realiza inversiones en educación, esta puede presentar un mayor nivel de desgaste de su capital humano previamente acumulado y pérdida de redes de contacto profesional. En consecuencia, deben presentar más significativas dificultades de reencontrar puestos de trabajo en el momento de recuperación del nivel de actividad económica. De esta forma, parece necesario el diseño de políticas o acciones que incentiven la formación continua de la mano de obra, la manteniendo en procesos de cualificación inclusive en los periodos de inactividad.

\section{Consideraciones finales}

Este artículo busca analizar las decisiones de los jóvenes entre 15 y 29 años de distribución del tiempo entre estudiar y pertenecer o no a la PEA. El análisis utiliza la base de datos de la Pesquisa Mensal de Emprego para seis regiones metropolitanas de Brasil, entre 2002 y 2015. Son utilizados dos métodos complementares de análisis: matrices de transición, para obtener la fracción de tiempo en que cada individuo permanece en determinada situación del mercado de trabajo, y un logit multinomial para auxiliar en la definición de como factores individuales alteran la entrada en cada situación.

Los resultados apuntan para mayores tasas de desocupación y de inactividad para los jóvenes del sexo femenino, sobre todo para aquellos entre 15 y 24 años, y una tendencia significativa de aumento a partir de 2012. El empleo de matrices de transición muestra una mayor fracción de tiempo en la inactividad para los jóvenes entre 15 y 17 años que estudian, siendo un resultado favorable para ambos los sexos. Por otro lado, la fracción de tiempo en que el joven permanece sin estudiar y sin buscar empleo, es decir, como ni-ni, es más expresiva para los jóvenes con edad entre 18 y 29 años, con aumento en el periodo más reciente.

Las estimaciones de un conjunto de modelos del tipo logit multinomial confirman estos resultados, además de indicar una dependencia temporal significativa de la posición en que se encuentra el joven en un periodo anterior, evidenciando dificultades para realizar transiciones, principalmente para aquellos que se encuentran fuera de la PEA y fuera del sistema educacional. Jóvenes negros y pardos, con 
menores niveles de escolaridad, residentes en las regiones metropolitanas de Salvador y de Rio de Janeiro presentan mayor probabilidad de no actuar en la PEA y de no estudiar al mismo tiempo. Factores macroeconómicos contribuyen para este comportamiento en el periodo reciente, cuando la economía brasileña presenta indicios de recesión, intensificada por problemas políticos, con reversión del escenario de avance registrado a lo largo del inicio del siglo XXI en el país.

En conclusión, el aumento de la proporción de jóvenes ni-ni y la expresiva dependencia temporal de esta condición apuntan para un escenario poco favorable para el mercado de trabajo brasileño. Este resultado puede tener impactos negativos en la vida de los jóvenes en un futuro de mediano y de largo plazo, compromete su capacidad productiva por la no acumulación de capital humano en una etapa crucial de su ciclo de vida.

\section{Referencias bibliográficas}

Becker, G. S. (1975), Human capital: a theoretical and empirical analysis, with special reference to education. 2. ${ }^{\mathrm{a}}$ ed. New York: Columbia University.

Blanchard, O. J. y Diamond, P. (1994), "Ranking, Unemployment Duration, and Wages", The Review of Economic Studies. Oxford University Press, 61(3), pp. 417-434. doi: 10.2307/2297897.

Borjas, G. (2008), Labour economics. 4. ${ }^{\text {a }}$ ed. New York: McGraw-Hill.

Cabanas, P., Komatsu, B. y Menezes-Filho, N. A. (2015), O crescimento da renda dos adultos e as escolhas dos jovens entre estudo e trabalho. 13. Cacciamali, M. C., Lima, T. T. y Tatei, F. (2015), "Determinantes da Duração do Desemprego no Brasil em Crises Econômicas", Revista Venezolana de Análisis de Coyuntura, pp. 187-209.

Camarano, A. A. (Organizadora) (2006), Transição para a vida adulta ou vida adulta em transição?, Instituto de Pesquisa Econômica Aplicada (Ipea). Brasília: Instituto de Pesquisa Econômica Aplicada (Ipea). Disponible en: http://repositorio.ipea.gov.br/handle/11058/3296 (Accedido: 25 de enero de 2019). 
Clark, K. B. y Summers, L. H. (1990), "Unemployment insurance and labor market transitions", en Understanding unemployment. Cambridge: MIT.

DIEESE, D. I. D. E. E. E. S. - (2008), Trajetórias da juventude nos mercados de trabalho metropolitanos: mudanças na inserção entre 1998 e 2007. São Paulo: DIEESE - Departamento Intersindical de Estatística e Estudos Socioeconômicos. Disponible en: https://www.dieese.org.br/livro/2008/2008pedjovensjuventude.pdf.

Geraci, A., Fabbri, D. y Monfardini, C. (2018), "Testing Exogeneity of Multinomial Regressors in Count Data Models: Does Two-stage Residual Inclusion Work?", Journal of Econometric Methods. De Gruyter, 7(1). doi: 10.1515/jem-2014-0019.

De Hoyos, R., Popova, A. y Rogers, H. (2016), Out of School and Out of Work: A Diagnostic of Ninis in Latin America. The World Bank (Policy Research Working Papers). doi: 10.1596/1813-9450-7548.

Kovrona, I., Lyon, S. y Rosati, F. C. (2013), NEET youth dynamics in Indonesia and Brazil: a cohort analysis. March.

Layard, R., Nickell, S. J. y Jackman, R. (2005), Unemployment: macroeconomic performance and the labour market. Oxford University Press.

Menezes-Filho, N. A., Cabanas, P. y Komatsu, B. (2013), A condição "nem-nem" entre os jovens é permanente? 7. São Paulo.

Mincer, J. y Jovanovic, B. (1979), Labor mobility and wages. 357. Cambridge, MA. doi: 10.3386/w0357.

Montgomery, J. D. (1991), "Social Networks and Labor-Market Outcomes: Toward an Economic Analysis", American Economic Review. American Economic Association, 81(5), pp. 1407-18.

Moreira, A. M. Z. y Queiroz, B. L. (2017), "A transição dos idosos no mercado de trabalho nas regiões metropolitanas brasileiras", en Encontro da ABET2. ABET. 
Nunes, D. U. et al. (2016), "Probabilidades de admissão e desligamento no mercado de trabalho brasileiro", Estudos Econômicos (São Paulo). Instituto de Pesquisas Econômicas da FEA-USP, 46(2), pp. 311-341. doi: 10.1590/0101-416146222dnb.

Pissarides, C. A. (1992), "Loss of Skill During Unemployment and the Persistence of Employment Shocks", The Quarterly Journal of Economics. Oxford University Press, 107(4), pp. 1371-1391. doi: $10.2307 / 2118392$.

Ribas, R. P. y Soares, S. S. D. (2008), "Sobre o painel da pesquisa mensal de emprego (PME) do IBGE". Brasília: Instituto de Pesquisa Econômica Aplicada (IPEA). Disponible en: https://www.econstor.eu/handle/10419/91005 (Accedido: 10 de octubre de 2018).

Samoilenko, A. y Carter, K. (2015), Economic Outcomes of Youth not in Education, Employment or Training (NEET), Treasury Working Paper Series. 15/01. New Zealand Treasury. Disponible en: https://ideas.repec.org/p/nzt/nztwps/15-01.html (Accedido: 16 de enero de 2019).

Simmons, R. (2008), "Raising the age of compulsory education in England: a neet solution?", British Journal of Educational Studies. Taylor \& Francis Group, 56(4), pp. 420-439. doi: 10.1111/j.14678527.2008.00417.x.

Wooldridge, J. M. (2007), Introdução à econometria: uma abordagem moderna. São Paulo: Thomson Learning.

Wooldridge, J. M. (2014), "Quasi-maximum likelihood estimation and testing for nonlinear models with endogenous explanatory variables", Journal of Econometrics. North-Holland, 182(1), pp. 226-234. doi: 10.1016/J.JECONOM.2014.04.020. 


\section{Anexo}

Tabla A.1. Contrastes de endogeneidad de los factores de status anterior - Mujeres. Fuente: elaboración propia con datos de la PME.

\begin{tabular}{|c|c|c|c|}
\hline & $\begin{array}{c}(1) \\
\text { PEA y Estudia }\end{array}$ & $\begin{array}{c}(3) \\
\mathrm{Ni}-\mathrm{Ni}\end{array}$ & $\begin{array}{c}(4) \\
\text { Fuera de la PEA y } \\
\text { Estudia }\end{array}$ \\
\hline Error (1) & 35,53 & $-168,78$ & 315,24 \\
\hline Error (3) & 127,98 & 37,67 & 184,42 \\
\hline Error (4) & $-220,33$ & 180,81 & $-615,93$ \\
\hline PEA y estudia en t- 1 & $0,33^{*}$ & $-1,38^{*}$ & $-1,81^{*}$ \\
\hline PEA y no estudia en $\mathrm{t}-1$ & $-2,58^{*}$ & $-1,74^{*}$ & $-4,56^{*}$ \\
\hline $\mathrm{Ni}-\mathrm{Ni}$ en $\mathrm{t}-1$ & $-2,54^{*}$ & $0,28^{*}$ & $-2,75^{*}$ \\
\hline Blancos & $0,34^{*}$ & $-0,09$ & $0,61^{*}$ \\
\hline Cónyuge & $-0,14^{* *}$ & $0,52^{*}$ & $-0,00$ \\
\hline Hijo & $0,64^{*}$ & $-0,19^{*}$ & $0,84^{*}$ \\
\hline Otros de la familia & $0,54^{*}$ & $-0,01$ & $0,73^{*}$ \\
\hline $4 / 7$ años de estudio & $-0,03$ & $-0,49^{*}$ & $0,17 * * *$ \\
\hline 8/10 años de estudio & $0,77^{*}$ & $-0,81^{*}$ & $0,38^{*}$ \\
\hline 11 o más años de estudio & $-0,51^{*}$ & $-1,25^{*}$ & $-1,61^{*}$ \\
\hline Edad & $-0,03^{*}$ & $-0,05^{*}$ & $-0,17^{*}$ \\
\hline Recife & 0,55 & $1,00^{*}$ & 0,40 \\
\hline Salvador & $0,43 *$ & $0,27 * *$ & $0,67 *$ \\
\hline Belo Horizonte & $0,20 * * *$ & $0,21^{* *}$ & 0,18 \\
\hline Rio de Janeiro & $-0,11$ & $0,61 * * *$ & 0,16 \\
\hline Porto Alegre & $0,31^{*}$ & 0,07 & $0,22^{*}$ \\
\hline 2004 & $-0,17$ & $-0,21$ & 0,30 \\
\hline 2005 & $-0,18$ & $-0,11$ & 0,43 \\
\hline 2006 & $-0,22$ & $-0,03$ & 0,15 \\
\hline 2007 & $-0,32$ & $-0,03$ & 0,10 \\
\hline 2008 & $-0,26$ & $-0,01$ & 0,26 \\
\hline 2009 & $-0,26$ & 0,05 & 0,31 \\
\hline 2010 & $-0,41$ & 0,06 & $-0,19$ \\
\hline 2011 & $-0,34$ & 0,10 & $-0,27$ \\
\hline 2012 & $-0,43$ & 0,00 & $-0,33$ \\
\hline 2013 & $-0,36$ & 0,05 & $-0,01$ \\
\hline 2014 & $-0,41$ & 0,31 & 0,07 \\
\hline 2015 & $-0,46$ & 0,28 & 0,15 \\
\hline Intercepto & $1,35^{* * *}$ & $1,40 * *$ & $4,62^{*}$ \\
\hline Pseudo - $\mathrm{R}^{2}$ & 0,3688 & & \\
\hline Número de obs. & 97548 & & \\
\hline $\mathrm{Chi}^{2}$ & 94938,28 & & \\
\hline Prob $>\mathrm{Chi}^{2}$ & 0,00 & & \\
\hline
\end{tabular}

$* * * \mathrm{p}<0.10,{ }^{* *} \mathrm{p}<0.05,{ }^{*} \mathrm{p}<0.01$ 
Tabla A.2. Contrastes de endogeneidad de los factores de status anterior - Varones. Fuente: elaboración propia con datos de la PME.

\begin{tabular}{|c|c|c|c|}
\hline & $\begin{array}{c}(1) \\
\text { PEA y Estudia }\end{array}$ & $\begin{array}{c}(3) \\
\mathrm{Ni}-\mathrm{Ni}\end{array}$ & $\begin{array}{c}\text { (4) } \\
\text { Fuera de la PEA y } \\
\text { Estudia }\end{array}$ \\
\hline Error (1) & $-74,53$ & 261,11 & $722,74^{*}$ \\
\hline Error (3) & $-36,51$ & $-165,37$ & 72,98 \\
\hline Error (4) & 172,41 & 20,61 & $-959,93 * *$ \\
\hline PEA y estudia en t- 1 & $0,16^{*}$ & $-1,50^{*}$ & $-1,95^{*}$ \\
\hline PEA y no estudia en $t-1$ & $-2,89 *$ & $-1,99^{*}$ & $-4,80 *$ \\
\hline $\mathrm{Ni}-\mathrm{Ni}$ en $\mathrm{t}-1$ & $-2,50 *$ & $-0,01$ & $-2,64^{*}$ \\
\hline Blancos & $0,42^{*}$ & $0,25^{*}$ & $0,82^{*}$ \\
\hline Cónyuge & $-0,27 * *$ & 0,15 & $-0,26$ \\
\hline Hijo & $0,53^{*}$ & $0,96^{*}$ & $0,86^{*}$ \\
\hline Otros de la familia & $0,32 *$ & $0,79^{*}$ & $0,67^{*}$ \\
\hline $4 / 7$ años de estudio & $-0,06$ & $-1,14^{*}$ & $-0,26^{*}$ \\
\hline 8/10 años de estudio & $0,56^{*}$ & $-1,59^{*}$ & 0,00 \\
\hline 11 o más años de estudio & $-0,35^{*}$ & $-1,51^{*}$ & $-1,38^{*}$ \\
\hline Edad & $-0,04^{*}$ & $-0,06^{*}$ & $-0,24^{*}$ \\
\hline Recife & 0,14 & $-0,81$ & $-0,63$ \\
\hline Salvador & 0,29 & 0,04 & $1,17^{*}$ \\
\hline Belo Horizonte & $0,22 * * *$ & 0,12 & $0,30 * *$ \\
\hline Rio de Janeiro & 0,06 & $-0,25$ & $-0,52$ \\
\hline Porto Alegre & $-0,04$ & $-0,12$ & 0,14 \\
\hline 2004 & $-0,03$ & $0,87^{*}$ & 0,27 \\
\hline 2005 & 0,01 & $0,63^{*}$ & 0,20 \\
\hline 2006 & 0,06 & 0,19 & $-0,22$ \\
\hline 2007 & 0,03 & 0,38 & $-0,30$ \\
\hline 2008 & 0,04 & $0,37 * *$ & $-0,16$ \\
\hline 2009 & 0,00 & 0,30 & $-0,58^{* * *}$ \\
\hline 2010 & $-0,02$ & 0,02 & $-0,80^{*}$ \\
\hline 2011 & 0,13 & 0,00 & $-1,63^{*}$ \\
\hline 2012 & 0,02 & 0,19 & $-0,38^{* *}$ \\
\hline 2013 & $-0,01$ & 0,12 & $-0,97^{*}$ \\
\hline 2014 & 0,03 & 0,11 & $-0,87^{* *}$ \\
\hline 2015 & 0,01 & $-0,28$ & $-0,79^{* * *}$ \\
\hline Intercepto & $-0,18$ & $-0,69$ & $5,50 *$ \\
\hline Pseudo - $\mathrm{R}^{2}$ & 0,3848 & & \\
\hline Número de obs. & 92789 & & \\
\hline $\mathrm{Chi}^{2}$ & 86759,76 & & \\
\hline Prob $>\mathrm{Chi}^{2}$ & 0,00 & & \\
\hline
\end{tabular}

$* * * \mathrm{p}<0.10,{ }^{* *} \mathrm{p}<0.05,{ }^{*} \mathrm{p}<0.01$ 